\author{
Professor Valentinas PODVEZKO, PhD \\ E-mail: valentinas.podvezko@vgtu.It \\ Department of Mathematical Statistics \\ Vilnius Gediminas Technical University, Vilnius, Lithuania \\ Professor Edmundas Kazimieras ZAVADSKAS, PhD \\ E-mail: edmundas.zavadskas@vgtu.lt \\ Laboratory of Operational Research, Faculty of Civil Engineering \\ Vilnius Gediminas Technical University, Vilnius, Lithuania \\ Principal Researcher Askoldas PODVIEZKO, PhD \\ E-mail: askoldas @ gmail.It \\ Agricultural Policy and Foreign Trade Division \\ Lithuanian Institute of Agrarian Economics, Vilnius, Lithuania
}

\title{
AN EXTENSION OF THE NEW OBJECTIVE WEIGHT ASSESSMENT METHODS CILOS AND IDOCRIW TO FUZZY MCDM
}

\begin{abstract}
Weights of criteria are playing a significant role in the wide range of MCDM (multiple criteria decision making) evaluation models. Results of evaluation significantly depend on magnitudes of weights as they proportionally transmit importance of each criterion to the final result of evaluation along with values of criteria placed in the decision matrix. There are two broad categories of methods for eliciting weights of criteria, subjective and objective. The former methods are based on opinions of experts, while the latter group of methods reflects the structure of data. The entropy method can be found within the most popular group of objective methods; it reflects the degree of diversification among values of criteria. Shortcomings of the entropy method are explained in the paper, especially the ones that are arising in the way of its extension to fuzzy MCDM. The authors propose extension of the earlier introduced criterion impact loss (CILOS) method to fuzzy MCDM that mitigate some shortcomings of the entropy. The latter method is based on the losses of values of criteria comparing to the ones that belong to the alternative with the best values. In the paper extension FIDOCRIW (Fuzzy Integrated Determination of Objective CRIteria Weights ) of the combination of both above-mentioned methods, entropy and CILOS, to fuzzy MCDM is proposed. Obstacles related to such extension are outlined and described. The paper provides a detailed explanation of the obstacles of extending the methods to fuzzy MCDM. Proposed in the paper FIDOCRIW method retains idea of the IDOCRIW method of combining the entropy with CILOS method. In contrast, the FIDOCRIW processes fuzzy numbers instead of real ones. This comprises uncertainty of data. The method allows to fully retain the fuzzy structure of the decision matrix along the full framework of the method. Resulting fuzzy weights allow to use the full scope of fuzzy MCDM methods, e.g. to comprise FIDOCRIW fuzzy weights with the fuzzy data that describe alternatives. Thus evaluation of alternatives in the environment with a degree of uncertainty can be performed.
\end{abstract}

Keywords: MCDM, criteria, objective weight, integrated determination, FCILOS, F-entropy, FIDOCRIW methods.

JEL Classification: C1, C01, C51, M11, 014, O18, D81

DOI: 10.24818/18423264/54.2.20.04 
Valentinas Podvezko, Edmundas Kazimieras Zavadskas, Askoldas Podviezko

\section{Introduction}

MCDM models, in general, are deterministic. This means that an exact input determines the exact output for a chosen MCDM method. The input for the majority of MCDM methods is a decision matrix $\mathbf{R}=\left\|r_{i j}\right\|$. It consists of data that characterizes evaluated alternatives $A_{l}, A_{2, \ldots}, A_{n}$ in terms of criteria $R_{l}, R_{2}, \ldots, R_{m}$. where $i=1,2, \ldots, n ; j=1,2, \ldots m ; m$ is the number of criteria while $n$ is the number of alternatives (Hwang andYoon, 1981; Mardani et al, 2017; Mardani et al, 2015; Vinogradova, 2019; Zavadskas and Turskis, 2011). Criteria are chosen depending on the specific problem faced by a decision-maker, the aim and objects of evaluation. Another input is the vector of weights $\boldsymbol{\Omega}=\left(\omega_{j}\right)$,which reflects importance of criteria in the scale of real numbers. These two inputs generate a determined outcome that ranks alternatives in the order of attractiveness. Such ranking is based on values of a cumulative criterion of a MCDM method that comprises values from the decision-matrix and weights.

The MCDM methods usually are lacking tools for dealing with uncertainty. Among non-fuzzy MCDM methods that have an effective inherent tool that deals with uncertainty the PROMETHEE method could be named. Choice of the proper preference function, either the multistage or the V-shape with indifference, could deal with the uncertainty in statistical data either by classifying data by intervals (multistage preference function case) or by curtailing extremes (V-shape with indifference preference function) (Podvezko and Podviezko, 2010).

Both MCDM methods and methods that allow to elicit weights are actively being actively developed. An extensive overview of MCDM methods is available in (Zavadskas and Podvezko, 2016). Weights of criteria form an essential part of MCDM methods considerably influencing the result of multiple criteria evaluation. Development of such methods is of great theoretical and practical importance. Currently there are many contemporary methods that allow to elicit weights. For example, Delphi method(Hwang and Lin, 1987), Expert judgment method (Zavadskas et al, 2012), Analytic Hierarchy Process (AHP) (Satty, 1980), Stepwise weight assessment ratio analysis (SWARA) (Kersuliene et al, 2010;Zavadskas and Podvezko, 2016, etc.). A more significant criterion will be assigned a larger weight and vice-versa. By definition the sum of weights should be equal to unity: $\sum_{j=1}^{m} \omega_{j}=1$. In such cases when the above does not hold, weights should be normalized.

Methods that elicit weights can be categorized into two categories: subjective and objective. The former, subjective, methods are based on opinions of experts, while the latter group reflects the structure of data. Even though they are presently more popular than the objective ones, the latter methods are being intensively developed and are gaining increasing recognition.

Objective methods analyze data in various ways. Among popular methods of eliciting weights are: LINMAP methodm (Hwang and Yoon, 1981); mathematical programming methods (Pekelman and Sen, 1974); methods that use 
An Extension of the New Objective Weight Assessment Methods CILOS and IDOCRIW to Fuzzy MCDM

correlation coeficient and standard deviation (Parfenova et al, 2016), least squares comparison (Wang and Lee, 2009) etc. (Zavadskas and Podvezko, 2016). The classic entropy method, another objective method, determines the degree of diversification among values of criteria (Hwang and Yoon, 1981). The authors proposed a weight-correction method based on Bayes method (Vinogradova et al, 2018). The entropy method can be distinguished as one of the most popular of objective method. In the authors' proposed the CILOS method (Zavadskas and Podvezko, 2016) structure of the data is analyzed in terms of losses of values of criteria. They are compared to the ones belonging to an alternative with the best values. These differeces directly influence weights (Cereska et al, 2018). The hybrid method IDOCRIW (Zavadskas and Podvezko, 2016) combines both entropy and CILOS methods. Shortcomings of the entropy revealed in (Zavadskas and Podvezko, 2016) are mitigated by the CILOS method.

The paper is devoted to developing of objective fuzzy-methods of eliciting weights; and to the analysis of the F-entropy method.

\section{Fuzzy numbers and operations with such numbers}

The fuzzy numbers were introduced by L. A. Zadeh (1965). The underlying theory well mingles with the theory of multiple criteria decisionmaking. It enhances it and extends to the realm of the uncertain data. Thus, a possibility to use intervals instead of precise data expressed in terms of a single number emerges. In addition, there is an option to assign the most probable value not even in the centre of the interval. Fuzzy numbers are often found in the theoretical and empirical literature from the realm of MCDM (Ecer, 2018; Hu et al, 2018; KutluGundogdu and Kahraman, 2019; Mardani et al, 2015; Rezaei and Ortt, 2013; Stanujkic et al, 2019) etc.

Triangle fuzzy numbers are used most often. Other popular shapes are trapezoids and analogs of the Gaussian curve. We will present illustrations with triangle fuzzy numbers below. Such numbers are defined by three numbers as follows: $\mathrm{M}=(l, m, u): l$ is the lower boundary; $m$ - is the most probable value; and $u$ - is the higher boundary. Naturally, $l \leq m \leq u$; usually $(l<m<u)$.

A fuzzy number is characterized by its triangle membership function

$$
\mu_{M}(x)=\left\{\begin{array}{l}
\frac{x-l}{m-l} \text { if } x \in[l, m] \\
\frac{x-u}{m-u} \text { if } x \in[m, u] \\
0 \text { if } x \notin[l, u] .
\end{array}\right.
$$

We will describe only the operations that are used in the paper. defined as:

The sum of two fuzzy numbers $M_{1}=\left(l_{1}, m_{1}, u_{1}\right)$ and $M_{2}=\left(l_{2}, m_{2}, u_{2}\right)$ is

$$
M_{1} \oplus M_{2}=\left(l_{l}+l_{2}, m_{1}+m_{2}, u_{1}+u_{2}\right)
$$

\section{DOI: 10.24818/18423264/54.2.20.04}


The difference of two fuzzy numbers $M_{1}=\left(l_{1}, m_{1}, u_{1}\right)$ and $M_{2}=\left(l_{2}\right.$, $\left.m_{2}, u_{2}\right)$ is defined as:

$$
M_{1} \ominus M_{2}=\left(l_{1}-l_{2}, m_{1}-m_{2}, u_{1}-u_{2}\right) ;
$$

The multiplication of two fuzzy numbers $M_{1}=\left(l_{1}, m_{1}, u_{1}\right)$ and $M_{2}=\left(l_{2}, m_{2}\right.$, $\left.u_{2}\right)$ is defined as:

$$
M_{1} \otimes M_{2}=\left(l_{1} l_{2}, m_{1} m_{2}, u_{1} u_{2}\right)
$$

The multiplicationby a number $\lambda$ is:

$\lambda \otimes M_{1}=(\lambda, \lambda, \lambda) \otimes\left(1_{1}, \mathrm{~m}_{1}, \mathrm{u}_{1}\right)=\left(\lambda \mathrm{l}_{1}, \lambda \mathrm{m}_{1}, \lambda \mathrm{u}_{1}\right) ;$ isdefined as:

The division of two fuzzy numbers $M_{l}=\left(l_{l}, m_{1}, u_{1}\right)$ and $M_{2}=\left(l_{2}, m_{2}, u_{2}\right)$

$$
M_{1} \div M_{2}=\left(l_{1} / u_{2}, m_{1} / m_{2}, u_{1} / l_{2}\right)
$$

The inverse of the fuzzy number $M_{1}=\left(l_{1}, m_{1}, u_{1}\right)$ is defined as:

$$
M_{1}^{-1}=\left(l_{1}, m_{1}, u_{1}\right)^{-1}=\left(1 / u_{1}, 1 / m_{1}, 1 / l_{1}\right) .
$$

The natural logarithm of the fuzzy number $M_{l}=\left(l_{l}, m_{l}, u_{l}\right)$ is defined as:

$$
\ln \left(M_{1}\right)=\left(\ln \left(l_{1}\right), \ln \left(m_{1}\right), \ln \left(u_{1}\right)\right)
$$

We can observe that the result of the above operations is, again, the set of three numbers with a corresponding membership function.

\section{Defuzzification of fuzzy numbers}

Defuzzification is a mapping of fuzzy numbers to the scale of real numbers. It is, of course, possible to apply standard MCDM methods after the defuzzification, but such option would lead to loss of important information about the inherent uncertainty, which can affect the result. Contrary, in fuzzy MCDM methods, values of criteria, weights, all intermediate results, as well as the final result, can be expressed in fuzzy numbers.

One of the most popular and simplest methods of defuzzification is the Center of Gravity (COG) method. Defuzzification is carried out using the following formula (Rezaei and Ortt, 2013):

$R=\frac{(u-l)+(m-l)}{3}+l=\frac{l+m+u}{3}$,

where $\mathrm{M}=(l, m, u)$.

\section{Objective fuzzy methods of eliciting weights}

Suppose the decision matrix with elements $\tilde{r}_{i j}=\left(r_{i j}^{L}, r_{i j}^{M}, r_{i j}^{U}\right)$ is provided. In order to apply MCDM methods we will need objective fuzzy weights denoted as $\widetilde{\Omega}=\left\|\widetilde{\omega}_{j}\right\|=\left(\widetilde{\omega}_{1}, \widetilde{\omega}_{2}, \ldots, \widetilde{\omega}_{m}\right)=\left\|\omega_{j}^{L}, \omega_{j}^{M}, \omega_{j}^{U}\right\|=$ $\left(\left(\omega_{1}^{L}, \omega_{1}^{M}, \omega_{1}^{U}\right),\left(\omega_{2}^{L}, \omega_{2}^{M}, \omega_{2}^{U}\right), \ldots,\left(\omega_{m}^{L}, \omega_{m}^{M}, \omega_{m}^{U}\right)\right), i=1, \ldots, n, j=1, \ldots, m$, 
An Extension of the New Objective Weight Assessment Methods CILOS and IDOCRIW to Fuzzy MCDM

where $m$ is the number of criteria, and $n$ is the number of alternatives. Some of such methods are presented below.

\subsection{Fuzzy entropy method F-Entropy}

The entropy method was proposed by Shannon (1948).

Entries of the normalized decision matrix are fuzzy numbers $\tilde{r}_{i j}=\left(r_{i j}^{L}\right.$, $\left.r_{i j}^{M}, r_{i j}^{U}\right), i=1, \ldots, n, j=1, \ldots, m$, where $m$-is the number of criteria, and $n$ - is the number of alternatives being evaluated.

Entropy weights are elicited in the following steps.

1. Values of criteria are normalized using the following formula:

$\tilde{s}_{i j}=\frac{\tilde{r}_{i j}}{\sum_{i=1}^{n} \tilde{r}_{i j}}=\left(\frac{r_{i j}^{L}}{\sum_{i=1}^{n} r_{i j}^{U}}, \frac{r_{i j}^{M}}{\sum_{i=1}^{n} r_{i j}^{M}}, \frac{r_{i j}^{U}}{\sum_{i=1}^{n} r_{i j}^{L}}\right)=\left(s_{i j}^{L}, s_{i j}^{M}, s_{i j}^{U}\right)$.

2. Entropy level of every criterion is calculated as follows:

$\tilde{E}_{j}=(-1 / \ln n) \sum_{i=1}^{n} \widetilde{r_{l \jmath}} \cdot \ln \widetilde{r_{l j}} \quad=\left(e_{j}^{L}, e_{j}^{M}, e_{j}^{U}\right)$, $(j=1,2, \ldots, m)$.

3. Non-normalized entropy weights are calculated using the following formula:

$\tilde{d}_{j}=1-\tilde{E}_{j}=\left(1-e_{j}^{L}, 1-e_{j}^{M}, 1-e_{j}^{U}\right)=\left(d_{j}^{L}, d_{j}^{M}, d_{j}^{U}\right)$

4. After normalization fuzzy weights are obtained:

$\widetilde{W}_{j}=\frac{\tilde{d}_{j}}{\sum_{j=1}^{m} \tilde{d}_{j}}=\left(\frac{d_{j}^{L}}{\sum_{j=1}^{m} d_{j}^{U}}, \frac{d_{j}^{M}}{\sum_{j=1}^{m} d_{j}^{M}}, \frac{r_{i j}^{U}}{\sum_{j=1}^{m} d_{j}^{L}}\right)=\left(W_{j}^{L}, W_{j}^{M}, W_{j}^{U}\right)$

A decision-maker should be aware about the following particularity of the entropy method for its effective use.

Proposition. Results of the F-entropy can be negative numbers.

Proof.

It is known that normalized entropy values $\frac{r_{i j}^{M}}{\sum_{i=1}^{n} r_{i j}^{M}}$ at the point $\mathrm{M}$ belong to the interval [0,1]: $0 \leq e_{j}^{M} \leq 1$.

Now, in the fuzzy case, at the point $U$, since

$\sum_{i=1}^{n} r_{i j}^{U}>\sum_{i=1}^{n} r_{i j}^{L}$ it follows that $\frac{r_{i j}^{U}}{\sum_{i=1}^{n} r_{i j}^{L}}>\frac{r_{i j}^{U}}{\sum_{i=1}^{n} r_{i j}^{U}}$. Therefore, entropy

$e_{j}^{U}=(-1 / \ln n) \sum_{i=1}^{n} \frac{r_{i j}^{U}}{\sum_{i=1}^{n} r_{i j}^{L}} \cdot \ln \frac{r_{i j}^{U}}{\sum_{i=1}^{n} r_{i j}^{L}}$ can be larger than $1, d_{j}^{U}=1-e_{j}^{U}$, and the weight $W_{j}^{U}$ will be negative.

There were numerous such cases encountered in practice. In Section 6 below an illustrative case-study is presented.

This particularity of the F-entropy method restricts usage of the method. The FCILOS method is free from such a shortcoming.

DOI: 10.24818/18423264/54.2.20.04 


\subsection{The fuzzy method of the criterion impact loss FCILOS}

The newly introduced method CILOS of the criterion impact loss assesses losses of each criterion against the alternative with the optimal (maximal or minimal) value. The logic, ideas and stages of the method were described in (Zavadskas and Podvezko, 2016).

At the first stage minimizing criteria are transformed to the maximizing ones using formula (14) to map the best value of a criterion to the largest value. Values of maximizing criteria remain unchanged.

$\bar{r}_{i j}=\frac{\min _{i} \tilde{r}_{i j}}{\tilde{r}_{i j}}=\frac{\left(\min _{i} r_{i j}^{L}, \min _{i} r_{i j}^{M}, \min _{i} r_{i j}^{U}\right)}{\left(r_{i j}^{L}, r_{i j}^{M}, r_{i j}^{U}\right)}=\left(\frac{\min _{i} r_{i j}^{L}}{r_{i j}^{U}}, \frac{\min _{i} r_{i j}^{M}}{r_{i j}^{M}}, \frac{\min _{i} r_{i j}^{U}}{r_{i j}^{L}}\right)$

$r_{i j}^{L} \neq 0, r_{i j}^{M} \neq 0, r_{i j}^{U} \neq 0$.

The new matrix is denoted as

$\widetilde{X}=\left\|\tilde{x}_{i j}\right\|=\left(x_{i j}^{L}, x_{i j}^{M}, x_{i j}^{U}\right)$.

Largest values of each criterion in each column of the matrix are found:

$\tilde{x}_{j}=\max _{i} \tilde{x}_{i j}=\left(\max _{i} x_{i j}^{L}, \max _{i} x_{i j}^{M}, \max _{i} x_{i j}^{U}\right)=\left(x_{k_{j}(L), j}^{L}, x_{k_{j}(M), j}^{M}, x_{k_{j}(U), j}^{U}\right)$, where $\left.k_{j}(L), k_{j}(M), k_{j}(U)\right)$ are numbers of rows of matrices $L, M, U$ of the $j$-th column corresponding to the largest elements.

The matrix $\widetilde{A}=\left\|\tilde{a}_{i j}\right\|=\left(a_{i j}^{L}, a_{i j}^{M}, a_{i j}^{U}\right)$ is consisting of values $\tilde{x}_{k_{j} j}$ of the $k_{j}$ th rows of the matrix $\widetilde{X}$ which correspond to maxima $\tilde{a}_{j j}$ of the $j$-th criterion : $\tilde{a}_{j j}=\tilde{x}_{j}=\left(x_{j}^{L}, x_{j}^{M}, x_{j}^{U}\right), \tilde{a}_{i j}=\tilde{x}_{k_{j} j}(i, j=1,2, \ldots, m ; m$ - the number of criteria).

Three square matrices AL, AM, AU are formed from elements of the matrix $\widetilde{A}$, which contain maximal values of each criterion on the main diagonal.

We note that the matrix $\widetilde{A}$ may contain identical rows in case several largest values were found in the same row that belong to the same alternative.

The matrices of relative losses $P^{L}, P^{M}$ and $P^{U}$ are formed.Their entries belong to the fuzzy matrix $\widetilde{P}=\left\|\tilde{p}_{i j}\right\|=\left(p_{i j}^{L}, P_{i j}^{M}, p_{i j}^{U}\right)$ with the following elements:

$\tilde{p}_{i j}=\frac{\tilde{x}_{j}-\tilde{a}_{i j}}{\tilde{x}_{j}}=\frac{\left(x_{j}^{L}-a_{i j}^{L}, x_{j}^{M}-a_{i j}^{M}, x_{j}^{U}-a_{i j}^{U}\right)}{\left(x_{j}^{L}, x_{j}^{M}, x_{j}^{U}\right)}=\left(\frac{x_{j}^{L}-a_{i j}^{L}}{x_{j}^{U}}, \frac{x_{j}^{M}-a_{i j}^{M}}{x_{j}^{M}}, \frac{x_{j}^{U}-a_{i j}^{U}}{x_{j}^{L}}\right)$

$\left(\tilde{p}_{i i}=0\right) \quad(i, j=1,2, \ldots, m)$.

Elements $\tilde{p}_{i j}$ of the matrix $\widetilde{P}$ contain relative losses of each criterion between values of alternatives and values of the alternative with the best $i$-th value. The method produces an increased weight for the criterion with a smaller loss while the resulting weight will appear to be smaller in the case if the loss is large. Thus the requirement of a criterion with larger losses to be less significant is fulfilled.

It was proved that (Mirkin, 1979): 
An Extension of the New Objective Weight Assessment Methods CILOS and IDOCRIW to Fuzzy MCDM

- in case losses of the $j$-th criterion increase $k$ times the corresponding weight will decrease $k$ times, while alterations of other values will not influence the $j$-th weight;

- the ratio $q_{i} / q_{j}$ between two weights $i$ and $j$ will not change if values of a criterion $l(l \neq i, l \neq j)$ are altered.

Following the second feature of the method the system (16) is created

$$
q_{i} p_{i j}=q_{j} p_{j i}
$$

which has $m(m-1) / 2$ equations with $m$ unknown variables. Thus it can be inconsistent. The system has the only solution when $m(m-1) / 2=m$, or when $m=3$.

Further, it was proved (Mirkin, 1979) that the system of linear equations (16) becomes consistent if the following equations are added:

$$
q_{i} \sum_{i=1}^{m} p_{i 1}=\sum_{j=1}^{m} q_{j} p_{i j} \text {. }
$$

The latter equations represent the idea that losses of the $i$-th criterion are equal to the sum of losses of all other criteria; they are compensated by the remaining criteria.

The system (16-17) can be re-written in the fuzzy form (18) using the vector of weights $\widetilde{q_{J}}=\left(q_{j}^{L}, q_{j}^{M}, q_{j}^{U}\right)(j=1,2, \ldots, m)$ as the unknown:

$$
\widetilde{\boldsymbol{F}} \boldsymbol{q}^{T}=0,
$$

where the matrix $\widetilde{\boldsymbol{F}}$ is:

$$
\widetilde{\boldsymbol{F}}=\left(\begin{array}{cccc}
-\sum_{i=1}^{m} \tilde{p}_{i 1} \tilde{p}_{12} & \ldots & \tilde{p}_{1 m} \\
\tilde{p}_{21} & -\sum_{i=1}^{m} \tilde{p}_{i 2} & \ldots & \tilde{p}_{2 m} \\
\ldots & & & \\
\tilde{p}_{m 1} \tilde{p}_{m 2} & \ldots & -\sum_{i=1}^{m} \tilde{p}_{i m}
\end{array}\right) .
$$

with entries $\tilde{p}_{i j}=\left(p_{i j}^{L}, P_{i j}^{M}, p_{i j}^{U}\right)$.

Three following determined systems of linear equations (20) are solved. $F^{L} \boldsymbol{q}^{\boldsymbol{T}}=0, F^{M} \boldsymbol{q}^{\boldsymbol{T}}=0, F^{U} \boldsymbol{q}^{\boldsymbol{T}}=0$.

Its solution provides fuzzy weights $\widetilde{q_{J}}=\left(q_{j}^{L}, q_{j}^{M}, q_{j}^{U}\right)$.

There are infinite number of solutions existing of a homogenous system of $m$ equations and $n$ unknowns (20).Ultimate weights are obtained by normalization.

DOI: $10.24818 / 18423264 / 54.2 .20 .04$ 


\subsection{FIDOCRIW method of hybrid objectives weights}

Using the idea of combining weights produced by the above-described methods with different inherent philosophy we can obtain combined weights $\widetilde{W}_{j}=\left(W_{j}^{L}, \quad W_{j}^{M}, W_{j}^{U}\right)$ obtained by the F-entropy method with weights $\widetilde{q_{J}}=\left(q_{j}^{L}, q_{j}^{M}, q_{j}^{U}\right)$ and the ones obtained by the FCILOS method. Hybrid fuzzy weights $\widetilde{\Omega_{j}}=\left(\omega_{j}^{L}, \omega_{j}^{M}, \omega_{j}^{U}\right)$ are obtained using the following multiplication (21):

$\widetilde{\Omega_{J}}=\frac{\widetilde{q_{J}} \otimes \widetilde{W}_{j}}{\sum_{j=1}^{m} \widetilde{q_{J}} \otimes \widetilde{W}_{j}}=\left(\frac{q_{j}^{L} W_{j}^{L}}{\sum_{j=1}^{n} q_{j}^{U} W_{j}^{U}} \frac{q_{j}^{M} W_{j}^{M}}{\sum_{j=1}^{n} q_{j}^{M} W_{j}^{M}}, \frac{q_{j}^{U} W_{j}^{U}}{\sum_{j=1}^{n} q_{j}^{L} W_{j}^{L}}\right)=\left(\omega_{j}^{L}, \omega_{j}^{M}, \omega_{j}^{U}\right)$.

The latter weights encompass the degree of diversification among values of criteria because of using the entropy. In addition to it, in (Zavadskas and Podvezko, 2016) it was demonstrated that shortcomings of the entropy method will be compensated by the CILOS method because there is a logical inverse relationship between losses related to other criteria and the degree of diversification.

\section{A case study involving the FCILOS, F-ENTROPY, and IDOCRIW methods}

We will apply the above-described fuzzy methods to the case-study described in Zavadskas and Podvezko, 2016. Thedata is presented in Table 1;it was a non-fuzzy case. We will compare both fuzzy and non-fuzzy cases and test stability of the methods.

We briefly describe the problem outlined in (Zavadskas and Podvezko, 2016). Some alternatives office building available for sale are considered by a company. There are four variants $A_{1}, A_{2}, A_{3}, A_{4}$ of office location. The offices are evaluated by the four attributes:

1) $R_{l}$ - price $(\$ 10,000)$,

2) $R_{2}$ office area $\left(\mathrm{m}^{2}\right)$,

3) $R_{3}$ - distance from home to work $(\mathrm{km})$,

4) $R_{4}$ - office location quality (in points).

The attributes $R_{2}$ and $R_{4}$ are maximizing, while $R_{l}$ and $R_{3}$ are minimizing. The data concerning office purchasing for a firm is presented in Table 1.

Table 1. Date of office purchasing

\begin{tabular}{|c|c|c|c|c|}
\hline \multirow{2}{*}{ Alternatives } & \multicolumn{4}{|c|}{ Attributes } \\
\cline { 2 - 5 } & $R_{1}$ & $R_{2}$ & $R_{3}$ & $R_{4}$ \\
\hline$A_{1}$ & 3.0 & 100 & 10 & 7 \\
\hline$A_{2}$ & 2.5 & 80 & 8 & 5 \\
\hline$A_{3}$ & 1.8 & 50 & 20 & 11 \\
\hline$A_{4}$ & 2.2 & 70 & 12 & 9 \\
\hline & $\min$ & $\max$ & $\min$ & $\max$ \\
\hline
\end{tabular}


An Extension of the New Objective Weight Assessment Methods CILOS and IDOCRIW to Fuzzy MCDM

The above matrix was transformed into the fuzzy one (Table 2).

Table 2.Fuzzy date of office purchasing

\begin{tabular}{|c|c|c|c|c|}
\hline \multirow{2}{*}{ Alternatives } & \multicolumn{4}{|c|}{ Attributes } \\
\cline { 2 - 5 } & $R_{1}$ & $R_{2}$ & $R_{3}$ & $R_{4}$ \\
\hline$A_{1}$ & $(2.95,3.0$, & $(96,100$, & $(9.5,10,11)$ & $(6.8,7,7.5)$ \\
& $3.03)$ & $103)$ & & \\
\hline$A_{2}$ & $(2.47,2.5$, & $(78,80,83)$ & $(7.5,8,9)$ & $(4.9,5,5.2)$ \\
& $2.55)$ & & & \\
\hline$A_{3}$ & $(1.77,1.8$, & $(49,50,52)$ & $(19,20,22)$ & $(10.8,11$ \\
& $2.85)$ & & & $11.3)$ \\
\hline$A_{4}$ & $(2.15,2.2$, & $(68,70,71)$ & $(11,12,14)$ & $(8.7,9,9.4)$ \\
& $2.23)$ & & & $\max$ \\
\hline & $\min$ & $\max$ & $\min$ & $\max$ \\
\hline
\end{tabular}

As it will be observed, results of calculations (Tables 3-9) at the central point $\mathrm{M}$ of the fuzzy data yield all intermediate results and weights exactly the same as in the previously calculated case with the non-fuzzy data (Zavadskasand Podvezko, 2016).

\subsection{Eliciting weights using the FCILOS method}

In accordance with the method of criterion impact loss (CILOS) all minimizing criteria should be transformed to the maximizing ones, for example, using formula (14). We have two minimizing criteria $R_{l}$ and $R_{3}$ in our case-study that need to be transformed. Transformed values are presented in Table 3. This is an optional visualization made solely for convenience purposes of the graphical observation of losses as they are essential part of the method.

Table 3. Transformation of fuzzy values of the minimizing criteria $R_{1}$ and $R_{3}$ to the maximizing ones

\begin{tabular}{|c|c|c|c|c|}
\hline Alternatives & $R_{1}$ & $R_{2}$ & $R_{3}$ & $R_{4}$ \\
\hline$A_{I}$ & $(0.584,0.6$, & $(75,100$, & $(0.682,0.8$, & $(4,7,9)$ \\
& $0.627)$ & $110)$ & $0.947)$ & \\
\hline$A_{2}$ & $(0.694,0.720$, & $(65,80,105)$ & $(0.843,1.0$, & $(3,5,8)$ \\
& $0.740)$ & & $1.200)$ & \\
\hline$A_{3}$ & $(0.957,1.0$, & $(40,5070)$ & $0.341,0.4,0.474)$ & $9,1114)$ \\
& $1.045)$ & & & \\
\hline$A_{4}$ & $(0.794,0.818$, & $(55,70,85)$ & $(0.536,0.667$, & $(7,9,12)$ \\
& $0.860)$ & & $0.818)$ & \\
\hline & $\max$ & $\max$ & $\max$ & $\max$ \\
\hline
\end{tabular}

DOI: $10.24818 / 18423264 / 54.2 .20 .04$ 
Valentinas Podvezko, Edmundas Kazimieras Zavadskas, Askoldas Podviezko Table 4.

Normalized by formula (10) values contained in Table 3 are presented in

Table 4. Normalized Fuzzy values of criteria

\begin{tabular}{|c|c|c|c|c|}
\hline Alternatives & $R_{I}$ & $R_{2}$ & $R_{3}$ & $R_{4}$ \\
\hline$A_{I}$ & $(0.178,0.191$, & $(0.311,0.333$, & $(0.198,0.279$, & $(0.204$, \\
& $0.207)$ & $0.354)$ & $0.396)$ & $0.219,0.240)$ \\
\hline$A_{2}$ & $(0.212,0.229$, & $(0.252,0.267$, & $(0.242,0.349$, & $(0.147$, \\
& $0.247)$ & $0.285)$ & $0.502)$ & $0.156,0.167)$ \\
\hline$A_{3}$ & $(0.292,0.319$, & $(0.159,0.167$, & $(0.099,0.140$, & $(0.323$, \\
& $0.345)$ & $0.179)$ & $0.198)$ & $0.344,0.362)$ \\
\hline$A_{4}$ & $(0.242,0.261$, & $(0.220,0.233$, & $(0.156,0.233$, & $(0.260$, \\
& $0.284)$ & $0.244)$ & $0.342)$ & $0.281,0.301)$ \\
\hline
\end{tabular}

The CILOS method at the subsequent step is applied to the lower and higher boundaries $\mathrm{L}$ and $\mathrm{U}$, and to the most probable - central value $\mathrm{M}$. We obtain the following resulting matrices $R N^{L}, R N^{M}$ and $R N^{U}$ :

$$
\begin{aligned}
R N^{L} & =\left(\begin{array}{llll}
0.178 & 0.311 & 0.198 & 0.204 \\
0.212 & 0.252 & 0.242 & 0.147 \\
0.292 & 0.159 & 0.099 & 0.329 \\
0.242 & 0.220 & 0.156 & 0.260
\end{array}\right), \\
R N^{M} & =\left(\begin{array}{llll}
0.191 & 0.333 & 0.279 & 0.219 \\
0.229 & 0.267 & 0.349 & 0.156 \\
0.319 & 0.167 & 0.140 & 0.344 \\
0.261 & 0.233 & 0.233 & 0.281
\end{array}\right), \\
R N^{U} & =\left(\begin{array}{llll}
0.207 & 0.354 & 0.396 & 0.240 \\
0.247 & 0.285 & 0.502 & 0.167 \\
0.345 & 0.179 & 0.198 & 0.362 \\
0.284 & 0.244 & 0.342 & 0.301
\end{array}\right) .
\end{aligned}
$$

Now the algorithm of the CILOS method is applied to the fuzzy case. The maximal values of each criterion (or each column) in each matrix $R N^{L}$, $R N^{M}$ and $R N^{U}$ are found. For example, in matrix $R N^{L}$ the maximal value of the criterion $R_{l}$ is 0.292 (3-rd row), the maximal value of the criterion $R_{2}$ is 0.311 (1-st row.), etc.

Then, the matrix $\widetilde{A}=\left\|\tilde{a}_{i j}\right\|=\left(a_{i j}^{L}, a_{i j}^{M}, a_{i j}^{U}\right)$ is compiled by forming separately matrices $\boldsymbol{A}^{\boldsymbol{L}}, \boldsymbol{A}^{\boldsymbol{M}}$ and $\boldsymbol{A}^{\boldsymbol{U}}$, from the rows found at the previous step. From the matrix $R N^{L}$ the 3-rd row, which corresponds to the maximal value of the 
An Extension of the New Objective Weight Assessment Methods CILOS and IDOCRIW to Fuzzy MCDM

criterion $R_{l}$, is taken. The row is placed to the first position in the matrix $A^{L}$. The second row of the matrix $\boldsymbol{A}^{\boldsymbol{L}_{\text {is }}}$ formed by taking the 1 -st row, which corresponds to the place of the maximal value of the criterion $R_{2}$, found in the row of the matrix $R N^{L}$, etc. Matrices $R N^{M}$ and $R N^{U}$ are formed similarly.

The resulting matrices appear to be as follows:

$$
\begin{aligned}
\boldsymbol{A}^{L} & =\left(\begin{array}{llll}
0.292 & 0.159 & 0.099 & 0.323 \\
0.178 & 0.311 & 0.198 & 0.204 \\
0.212 & 0.252 & 0.242 & 0.147 \\
0.292 & 0.159 & 0.099 & 0.323
\end{array}\right), \\
\boldsymbol{A}^{\boldsymbol{M}} & =\left(\begin{array}{llll}
0.319 & 0.167 & 0.140 & 0.344 \\
0.191 & 0.333 & 0.279 & 0.219 \\
0.229 & 0.267 & 0.349 & 0.156 \\
0.319 & 0.167 & 0.140 & 0.344
\end{array}\right), \\
\boldsymbol{A}^{U} & =\left(\begin{array}{llll}
0.345 & 0.179 & 0.197 & 0.362 \\
0.207 & 0.354 & 0.396 & 0.240 \\
0.247 & 0.285 & 0.502 & 0.167 \\
0.345 & 0.179 & 0.198 & 0.362
\end{array}\right) .
\end{aligned}
$$

The matrices of relative losses $\boldsymbol{P}^{L}, \boldsymbol{P}^{\boldsymbol{M}}$ and $\boldsymbol{P}^{U}$ are formed using the following formula:

$$
\begin{aligned}
& \tilde{p}_{i j}=\frac{\tilde{x}-\tilde{a}_{i j}}{\tilde{x}}=\left(p_{i j}^{L}, P_{i j}^{M}, p_{i j}^{U}\right)\left(\tilde{p}_{i i}=0\right) \text {. } \\
& \boldsymbol{P}^{L}=\left(\begin{array}{cccc}
0 & 0.430 & 0.285 & 0 \\
0.329 & 0 & 0.088 & 0.331 \\
0.232 & 0.165 & 0 & 0.488 \\
0 & 0.430 & 0.285 & 0
\end{array}\right) \text {, } \\
& \boldsymbol{P}^{M}=\left(\begin{array}{cccc}
0 & 0.500 & 0.600 & 0 \\
0.400 & 0 & 0.200 & 0.364 \\
0.280 & 0.200 & 0 & 0.545 \\
0 & 0.500 & 0.600 & 0
\end{array}\right) \text {, } \\
& \boldsymbol{P}^{U}=\left(\begin{array}{cccc}
0 & 0.564 & 1.253 & 0 \\
0.473 & 0 & 0.436 & 0.377 \\
0.335 & 0.221 & 0 & 0.605 \\
0 & 0.564 & 1.253 & 0
\end{array}\right) \text {. }
\end{aligned}
$$


Using formulae (16-18) the matrices of the homogeneous $F^{L}, F^{M}$ and $F^{U}$ are formed:

$$
\begin{aligned}
& F^{L}=\left(\begin{array}{cccc}
-0.561 & 0.4300 .285 & 0.000 \\
0.329 & -1.024 & 0.088 & 0.331 \\
0.232 & 0.165 & -0.659 & 0.488 \\
0.000 & 0.4300 .285 & -0.818
\end{array}\right), \\
& F^{M}=\left(\begin{array}{cccc}
-0.680 & 0.5000 .600 & 0.000 \\
0.400 & -1.200 & 0.200 & 0.364 \\
0.280 & 0.200 & -1.400 & 0.545 \\
0.000 & 0.5000 .600 & -0.909
\end{array}\right), \\
& F^{U}=\left(\begin{array}{cccc}
-0.809 & 0.5641 .253 & 0 \\
0.473 & -1.349 & 0.436 & 0.377 \\
0.335 & 0.201 & -2.944 & 0.605 \\
0 & 0.564 & 1.253 & -0.981
\end{array}\right)
\end{aligned}
$$

In the main diagonal of each matrix $F$ the corresponding element $f_{i i}$ represents the total loss of the $i$-th criterion, which has a direct influence on the weights. For example, observation of losses in the main diagonal of the matrix $F^{L}$ suggests that the criterion $R_{2}$ at the point $\mathrm{L}$ will have a smaller weight because of the larger losses, while criteria $R_{l}$ and $R_{3}$ will have the larger weight as it can be seen in Table 10. Similar observations are valid for matrices $F^{M}$ and $F^{U}$.

The vector of weights $\widetilde{q_{J}}=\left(q_{j}^{L}, q_{j}^{M}, q_{j}^{U}\right)$ is found by normalizing values of solutions of systems of the following equations:

$$
F^{L} \boldsymbol{q}^{\boldsymbol{T}}=0, F^{M} \boldsymbol{q}^{\boldsymbol{T}}=0, F^{U} \boldsymbol{q}^{\boldsymbol{T}}=0 .
$$

The values of the resulting vector of weights are presented in Table 5 .

Table 5. FCILOS weights of criteria

\begin{tabular}{|l|l|l|l|l|}
\hline & \multicolumn{1}{|c|}{$\widetilde{q_{1}}$} & \multicolumn{1}{|c|}{$\widetilde{q_{2}}$} & \multicolumn{1}{c|}{$\widetilde{q_{3}}$} & $\widetilde{q_{4}}$ \\
\hline $\mathrm{L}$ & 0.300 & 0.189 & 0.305 & 0.206 \\
\hline $\mathrm{M}$ & 0.334 & 0.220 & 0.196 & 0.250 \\
\hline $\mathrm{U}$ & 0.351 & 0.242 & 0.118 & 0.289 \\
\hline Average & 0.328 & 0.217 & 0.206 & 0.211 \\
\hline
\end{tabular}

Because of the normalization, the requirement of the definition of weights is fulfilled: the sum of averages is equal to unity.

The largest weight obtained by the criterion impact loss method has the 1st criterion $R_{l}$. It can be observed that its total losses are smallest at all three points $\mathrm{L}, \mathrm{M}$ and $\mathrm{U}$. 
An Extension of the New Objective Weight Assessment Methods CILOS and IDOCRIW to Fuzzy MCDM

\subsection{Eliciting weights using the F-entropy method}

Values of criteria were taken from Table 3 and normalized by formula (10). The result of normalization is presented in Table 6.

Table 6.Normalized values of criteria

\begin{tabular}{|c|c|c|c|c|}
\hline Alternatives & $R_{1}$ & $R_{2}$ & $R_{3}$ & $R_{4}$ \\
\hline$A 1$ & $(0.305,0.316$, & $(0.311,0.333$, & $(0.170,0.200,0.234)$ & $(0.204,0.219$, \\
& $0.324)$ & $0.354)$ & & $0.240)$ \\
\hline$A 2$ & $(0.256,0.263$, & $(0.252,0.267$, & $(0.134,0.160,0.191)$ & $(0.147,0.156,0.167)$ \\
& $0.273)$ & $0.285)$ & & \\
\hline$A 3$ & $(0.183,0.189$, & $(0.159,0.167$, & $(0.339,0.400,0.468)$ & $(0.323,0.344,0.362)$ \\
& $0.198)$ & $0.179)$ & & \\
\hline$A 4$ & $(0.223,0.232$, & $(0.220$, & $(0.196,0.240,0.298)$ & $(0.260,0.281,0.301)$ \\
& $0.239)$ & $0.233,0.244)$ & & \\
\hline
\end{tabular}

The fuzzy entropy is calculated using formula (11). Results are presented in Table 7.

Table 7. Fuzzy values of entropy

\begin{tabular}{|c|c|c|c|c|}
\hline & $R_{I}$ & $R_{2}$ & $R_{3}$ & $R_{4}$ \\
\hline $\mathbf{L}$ & 0.978 & 0.964 & 0.906 & 0.953 \\
\hline $\mathbf{M}$ & 0.988 & 0.979 & 0.955 & 0.971 \\
\hline $\mathbf{U}$ & 0.997 & 0.994 & 0.990 & 0.989 \\
\hline
\end{tabular}

The formula (13) is applied only in in the case if all fuzzy entropy weights are positive. This may not always be the case because at the point $U$ the formula of the fuzzy normalization is $\frac{r_{i j}^{U}}{\sum_{i=1}^{n} r_{i j}^{L}}$. Value of the entropy can be thus larger than 1 that creates the negative weight, as it will happen in the case-study that is described below.

Non-normalized entropy weights are calculated using the following formula at each point $\mathrm{L}, \mathrm{M}$, and $\mathrm{U}$ :

$\tilde{d}_{j}=1-\tilde{E}_{j}$

The result of calculations is presented in Table 8 .

Table 8. Non-normalized $F$-entropy weights

\begin{tabular}{|c|c|c|c|c|}
\hline & $R_{1}$ & $R_{2}$ & $R_{3}$ & $R_{4}$ \\
\hline $\mathbf{L}$ & 0.022 & 0.036 & 0.094 & 0.047 \\
\hline $\mathbf{M}$ & 0.012 & 0.021 & 0.045 & 0.029 \\
\hline $\mathbf{U}$ & 0.003 & 0.006 & 0.010 & 0.011 \\
\hline
\end{tabular}


Valentinas Podvezko, Edmundas Kazimieras Zavadskas, Askoldas Podviezko

Normalized entropy weights are calculated. Results are presented in Table 9.

Table 9. F-entropy weights

\begin{tabular}{|c|c|c|c|c|}
\hline & $R_{I}$ & $R_{2}$ & $R_{3}$ & $R_{4}$ \\
\hline $\mathbf{L}$ & 0.109 & 0.183 & 0.471 & 0.237 \\
\hline $\mathbf{M}$ & 0.115 & 0.198 & 0.418 & 0.269 \\
\hline $\mathbf{U}$ & 0.094 & 0.211 & 0.325 & 0.370 \\
\hline
\end{tabular}

Resulting values at the point $\mathrm{M}$ appear to be identical to the already calculated ones in(Zavadskas and Podvezko, 2016).

\subsection{Eliciting weights using the F-IDOCRIW method}

The weights obtained using the FCILOS (Table 5) and F-entropy (Table 9) methods are now combined into cumulative fuzzy weights. For the purpose of convenience, fuzzy weights previously obtained using the FCILOS and F-entropy methods are presented in Table 10. It is worth noting that $q_{j}^{L}<q_{j}^{M}<q_{j}^{U}$ (Table $10)$.

Table 10. FCILOS and F-entropy weights

\begin{tabular}{|c|c|c|c|c|c|}
\hline \multicolumn{2}{|c|}{} & $R_{1}$ & $R_{2}$ & $R_{3}$ & $R_{4}$ \\
\hline \multirow{2}{*}{$\mathrm{L}$} & CILOS, $q_{j}^{L}$ & 0.300 & 0.189 & 0.118 & 0.206 \\
\cline { 2 - 6 } & Entropy, $W_{j}^{L}$ & 0.094 & 0.183 & 0.325 & 0.237 \\
\hline \multirow{2}{*}{$\mathrm{M}$} & $\mathrm{CILOS}, q_{j}^{M}$ & 0.334 & 0.220 & 0.196 & 0.250 \\
\cline { 2 - 6 } & Entropy, $W_{j}^{M}$ & 0.109 & 0.198 & 0.418 & 0.269 \\
\hline \multirow{2}{*}{$\mathrm{U}$} & CILOS, $q_{j}^{U}$ & 0.351 & 0.242 & 0.305 & 0.289 \\
\cline { 2 - 6 } & Entropy, $W_{j}^{U}$ & 0.115 & 0.211 & 0.471 & 0.370 \\
\hline
\end{tabular}

Results obtained after combining the FCILOS and F-entropy weights by formula (21) are presented in Table 11 as hybrid weights of the FIDOCRIW method.

Table 11. Hybrid weights obtained using the FIDOCRIW method

\begin{tabular}{|c|c|c|c|c|}
\hline & $R_{I}$ & $R_{2}$ & $R_{3}$ & $R_{4}$ \\
\hline $\mathbf{L}$ & 0.0825 & 0.1011 & 0.1121 & 0.1428 \\
\hline $\mathbf{M}$ & 0.1589 & 0.1901 & 0.3575 & 0.2935 \\
\hline $\mathbf{U}$ & 0.2692 & 0.3405 & 0.9580 & 0.7131 \\
\hline Average weights & 0.1702 & 0.2106 & 0.4757 & 0.3931 \\
\hline
\end{tabular}

DOI: $10.24818 / 18423264 / 54.2 .20 .04$ 
Hybrid weights compensate shortcomings of entropy method as there is a logical inverse relationship between losses related to other criteria and the degree of diversification (Zavadskas and Podvezko, 2016). Extension of the IDOCRIW to fuzzy MCDM allows to use the weights in fuzzy MCDM methods. It is important as weights form an essential part of such methods.

\section{Problems to the usage of the F-entropy method}

It is known that values of the entropy are lying within the interval from 0 to $1 ;\left(0 \leq E_{j} \leq 1(j=1,2, \ldots, m)\right.$ ) (Hwang and Yoon, 1981;Zavadskas and Podvezko, 2016). Consequently, non-normalized values of entropy weights $d_{j}=1-E_{j}$ are nonnegative and entropy weights $W_{j}=\frac{d_{j}}{\sum_{j=1}^{m} d_{j}}$ are also non-negative. Fuzzy normalization $\frac{r_{i j}^{U}}{\sum_{i=1}^{n} r_{i j}^{L}}$ at the point $\mathrm{U}$ could create the normalized value larger than one. And the fuzzy entropy method could produce negative weights, which is not acceptable. criterion

For example, take the following altered (increased) values of the 1-st

$(2.8,3.0,3.3)$

$(2.4,2.5,2.7)$

$(1.7,1.8,2.1)$

$(2.0,2.2,2.5)$

Value of entropy in this case is $e_{1}^{U}=1.029>1$.It produces a negative weight. In such cases weights could be determined completely in the fuzzy space by the FCILOS method. In addition, weights can be elicited from experts.

\section{Conclusions}

Uncertainty of data should have influence on results of MCDM evaluation. Uncertainty of data could be dealt with using fuzzy numbers and fuzzy methods.

Objective weights that reflect the structure of data can be obtained using objective methods of eliciting weights from data. The popular entropy method assesses dominance of some criteria over other. The degree of dominance of the criteria with the best values influences weights of corresponding criteria. The method has shortcomings that are described in the paper; it may introduce distortions to the results of the MCDM evaluation as well.

The criterion impact loss (CILOS) method accounts losses of values of corresponding criteria comparing to the ones that belong to the alternative with the best values. The method compensates shortcomings of the entropy method, described in the paper.

DOI: $10.24818 / 18423264 / 54.2 .20 .04$ 
Valentinas Podvezko, Edmundas Kazimieras Zavadskas, Askoldas Podviezko

F-entropy weights at the point $U$ may attain negative values thus creating limitations to the usage of the method. The FCILOS method is free from such limitations.

The FIDOCRIW method combines two objective methods of eliciting weights, namely the F-entropy and FCILOS, while mitigating shortcomings of the F-entropy method appearing when the method is used in the fuzzy space.

Fuzzy weights allow to use the complete fuzzy structure of the fuzzy decision matrix; to use fuzzy MCDM methods; to combine subjective and objective fuzzy weights to hybrid weights; and to evaluate alternatives in the environment of uncertainty.

\section{REFERENCES}

[1] Cereska, A., Podviezko, A., Zavadskas, E.K. (2018), Assessment of Different Metal Screw Joint Parameters by Using Multiple Criteria Analysis Method. METALS 8(5);

[2] Ecer, F. (2018), An Integrated Fuzzy AHP and ARAS Model to Evaluate

Mobile Banking Service. Technological and Economic Development of Economy 84(2), 670-695;

[3] Hu, S.-K., Liou, J.L., Chuang, Y.-C., Tzeng, G.-H. (2018), New Hybrid FMADM Model for Mobile Commerce Improvement. Technological and Economic Development of Economy 24(5), 1801-1828;

[4] Hwang, C.L., Lin, M.J. (1987), Group Decision Making under Multiple Criteria: Methods and Applications; Berlin, Springer-Verlag;

[5] Hwang, C. L., Yoon, K. (1981), Multiple Attribute Decision Making Methods and Applications: A State-of-the-Art Survey; Berlin, Hedelberg: Springer;

[6] Kersuliene, V., Zavadskas, E.K., Turskis, Z. (2010), Selection of Rational

Dispute Resolution Method by Applying New Step-Wise Weight Assessment

Ratio Analysis (SWARA). Journal of Business Economics and Management 11(2), 243-258;

[7] Kutlu Gundogdu, F., Kahraman, C. (2019), Extension of WASPAS with Spherical Fuzzy Sets.Informatica 30(2), 269-292;

[8] Mardani, A., Jusoh, A., Zavadskas, E.K. (2015), Fuzzy Multiple Criteria Decision-Making Techniques andApplications - Two Decades Review from 1994 to 2014. Expert Systems with Applications 42(8), 4126-4148;

[9] Mardani, A., Zavadskas, E. K., Khalifah, Z., Zakuan, N., Jusoh, A., Nor, K., Khoshnoudi, M. (2017), A Review of Multi-criteria Decision-making Applications to Solve Energy Management Problems: Two Decades from 1995 to 2015. Renewable Sustainable Energy Reviews 71, 216-256;

[10] Mardani, A., Jusoh, A., Md Nor, K., Khalifah, Z., Zakwan, N., Valipour, A. (2015), Multiple Criteria Decision-making Techniques and their Applications -A Review of the Literature from 2000 to 2014. Economic Research-

EkonomskaIstraživanja 28(1), 516-571;

DOI: 10.24818/18423264/54.2.20.04 
An Extension of the New Objective Weight Assessment Methods CILOS and IDOCRIW to Fuzzy MCDM

[11] Mirkin, B.G. (1979), Group Choice; Winston\&Sons: Washington, DC, USA; [12] Parfenova, L., Pugachev, A., Podviezko, A. (2016), Comparative Analysis of Tax Capacity in Regions of Russia. Technological and Economic Development of Economy 22(6), 905-925;

[13] Pekelman, D., Sen, S.K. (1974), Mathematical Programming Models for the Determination of Attribute Weight. Management Science 20(8), 1217-1229;

[14] Podvezko, V., Podviezko, A. (2010), Dependence of Multi-criteria Evaluation Result on Choice of Preference Functions and their Parameters. Technological and Economic Development of Economy 16 (1), 143-158; [15] Rezaei, J., Ortt, R. (2013), Multi-criteria Supplier Segmentation Using a Fuzzy Preference Relations Based AHP. European Journal of Operational Research. 225(1), 75-84;

[16] Saaty, T.L. (1980), The Analytic Hierarchy Process; New York, McGraw Hill;

[17] Shannon, C. E. (1948), A Mathematical Theory of Communication. The Bell System Technical Journal 27, 379-423, 623-656;

[18] Stanujkic, D., Karabasevic, D., Zavadskas, E.K., Smarandache, F., Brauers, W.K.M. (2019), A Bipolar Fuzzy Extension of the MULTIMOORA Method.Informatica 30(1), 135-152;

[19] Vinogradova, I. (2019), Multi-Attribute Decision-Making Methods as a Part of Mathematical Optimization. Mathematics 7, 1-21;

[20] Vinogradova, I., Podvezko, V., Zavadskas, E.K. (2018), The Recalculation of the Weights of Criteria in MCDM Methods Using the Bayes Approach. Symmetry, 10(6):205;

[21] Wang, T.C., Lee, H.D. (2009), Developing a Fuzzy TOPSIS Approach Based on Subjective Weights and Objective Weights. Expert Systems with Applications 36(5), 8980-8985;

[22] Zadeh, L.A. (1965), Fuzzy Sets.Information and Control 8, 338-353;

[23] Zavadskas, E.K., Turskis, Z. (2011), Multiple Criteria Decision Making (MCDM) Methods in Economics: An Overview. Technological and Economic Development of Economy 17(2), 397-427;

[24] Zavadskas, E.K.; Podvezko, V. (2016), Integrated Determination of Objective Criteria Weights in MCDM.International Journal of Information Technology \& Decision Making 15(2), 267-283;

[25] Zavadskas, E.K., Vainiūnas, P., Turskis, Z., Tamošaitienè, J. (2012), Multiple Criteria Decision Support System for Assessment of Projects Managers in Construction.International Journal of Information Technology \& Decision Making 11(2), 501-520.

DOI: 10.24818/18423264/54.2.20.04 